\title{
Conformal Field Theories in Fractional Dimensions
}

\author{
Sheer El-Showk, ${ }_{1}^{1,2}$ Miguel Paulos, ${ }^{3}$ David Poland, ${ }^{4}$ Slava Rychkov, ${ }^{2,5}$ David Simmons-Duffin, ${ }^{6}$ and Alessandro Vichi ${ }^{7}$ \\ ${ }^{1}$ Institut de Physique Théorique CEA Saclay, CNRS-URA 2306, 91191 Gif sur Yvette, France \\ ${ }^{2}$ CERN, Theory Division, CH-1211 Geneva 23, Switzerland \\ ${ }^{3}$ Department of Physics, Brown University, Box 1843, Providence, Rhode Island 02912-1843, USA \\ ${ }^{4}$ Department of Physics, Yale University, New Haven, Connecticut 06520, USA \\ ${ }^{5}$ Laboratoire de Physique Théorique, École Normale Supérieure, Paris France and Faculté de Physique, \\ Université Pierre et Marie Curie, Paris, France \\ ${ }^{6}$ School of Natural Sciences, Institute for Advanced Study, Princeon, New Jersey 08540, USA \\ ${ }^{7}$ Theoretical Physics Group, Ernest Orlando Lawrence Berkeley National Laboratory and Center for Theoretical Physics, \\ University of California, Berkeley, California 94720, USA \\ (Received 16 December 2013; published 7 April 2014)
}

\begin{abstract}
We study the conformal bootstrap in fractional space-time dimensions, obtaining rigorous bounds on operator dimensions. Our results show strong evidence that there is a family of unitary conformal field theories connecting the 2D Ising model, the 3D Ising model, and the free scalar theory in 4D. We give numerical predictions for the leading operator dimensions and central charge in this family at different values of $D$ and compare these to calculations of $\phi^{4}$ theory in the $\varepsilon$ expansion.
\end{abstract}

DOI: 10.1103/PhysRevLett.112.141601

PACS numbers: $11.25 . \mathrm{Hf}$

Introduction.-The past few years have seen great progress in our understanding of conformal field theories (CFTs), particularly in three and four dimensions. The numerical analyses performed in [1-15] have clearly demonstrated that the "conformal bootstrap" constraints of unitarity and crossing symmetry [16,17] impose severe constraints on CFTs. Moreover, certain special theories (such as the 3D Ising model) appear to saturate these constraints. On the other hand, following recent advances in our understanding of conformal blocks [11,14,18-23] as well as analytic studies of the bootstrap [24-26], it has become transparent that the space-time dimension is simply a parameter which enters the bootstrap constraints. An analytic continuation to noninteger space-time dimension can be done in a completely straightforward way.

It is then natural to ask, does crossing symmetry have anything to say about the space of CFTs in noninteger dimensions? Can we find a family of solutions to the crossing symmetry constraint that interpolates between the 2D Ising model, the 3D Ising model, and the 4D free scalar? The purpose of this Letter is to start addressing these questions.

CFT in fractional dimensions. - The notion of noninteger dimensions is not new to quantum field theory. A widely used method to regularize the perturbative expansion of quantum field theories is dimensional regularization-analytically continuing Feynman integrals to noninteger dimensions. In this case, the analytic continuation is just a computational trick. Wilson and Fisher $[27,28]$ were the first to use such a continuation to connect theories living in different integer dimensions. They focused on $\phi^{4}$ theory in $D<4$ dimensions, which for $\varepsilon=4-D \ll 1$ has a weakly coupled infrared fixed point. Analytically continuing this family of fixed points to $\varepsilon=1$ and 2 should give, they argued, the infrared fixed point of the 3D and 2D Ising models. This observation is by now widely accepted and became the basis of the $\varepsilon$ expansion technique for computing the critical exponents of strongly coupled models. The results of the $\varepsilon$ expansion agree well with other approximation schemes, Monte Carlo simulations, and exact results when available. This strongly suggests that the basic idea is correct, in spite of the fact that it has never been justified beyond perturbation theory, and even a proper definition of what it means to have a field theory in noninteger $D$ has not been given.

In this Letter, we will provide new evidence for the existence of a line of fixed points interpolating between two and four dimensions which reduces to the Wilson-Fisher family for $4-D \ll 1$. Unlike in previous work, our analytic continuation is nonperturbative. It is defined by using the conformal symmetry of fixed points. Recall that the free 4D scalar theory, critical 2D Ising model, and, presumably, critical 3D Ising model possess such a symmetry, and we will assume that it survives for noninteger $D$ [29]. In integer dimensions, conformal symmetry leads to well-known constraints on correlation functions of local operators. For example, it fixes the correlator of four scalar operators up to a function $g(u, v)$ of conformal cross ratios. Since the number of independent cross ratios is the same (two) for any integer $D \geq 2$, it is natural to take the function $g(u, v)$ as the starting point for the analytic continuation. Recall that this function can be decomposed by using the operator product expansion (OPE) into a sum of conformal blocks corresponding to the exchanged operators. Inequivalent decomposition channels must produce the same four-point function, implying a crossing symmetry ("bootstrap") constraint. Furthermore, the conformal blocks are eigenfunctions of the quadratic Casimir operator, which 
depends on the space-time dimension $D$ analytically, so its eigenvalue equation can be solved by treating $D$ as a free parameter. We take the decomposition of the function $g(u, v)$ into analytically continued conformal blocks, together with the crossing symmetry constraint, as a definition of what it means to have conformal symmetry consistent with the OPE in noninteger dimensions.

Tracking Ising from $2 D$ to $4 D$.-Following the logic of [1], we can place rigorous upper bounds on the dimension $\Delta_{\epsilon}$ of the first nontrivial scalar operator $\epsilon$ entering the OPE of the lowest dimension operator $\sigma$ with itself:

$$
\sigma \times \sigma \sim 1+\epsilon+\cdots
$$

This is done by formulating the bootstrap constraints on the four-point function $\langle\sigma \sigma \sigma \sigma\rangle$ as a linear program and solving it numerically by using the simplex method. For a given value of $\Delta_{\sigma}$, the linear program has no solution if $\Delta_{\epsilon}$ is sufficiently large. The details of our methodology will be elaborated on in a future publication [30]. Notice that the only representations of $S O(D)$ that can occur in the conformal block decomposition of this four-point function are symmetric traceless tensors of rank $\ell=0,2,4, \ldots$. We analytically continue conformal blocks to noninteger $D$ separately for each $\ell$. We evaluate the blocks and their derivatives by using the expansion in radial coordinates [21], by the algorithm described in [22].
As first seen in $[2,11]$, the upper bound on $\Delta_{\epsilon}$ as a function of $\Delta_{\sigma}$ shows a sharp change of slope in both 2D and 3D. Within errors, the locations of these "kinks" agree with the known dimensions in the 2D and 3D Ising models. If we were to interpolate between these results by varying $D$, we would expect the position of the kink to evolve until it converges upon the free scalar theory in $4 \mathrm{D}$, where no kink has been observed. One might then hypothesize that for some as yet unknown but likely fundamental reason the critical points of the Ising universality class lie exactly at the kink determined with the best possible accuracy.

This intuition is indeed borne out by our analysis, summarized in Fig. 1, where we show upper bounds on $\Delta_{\epsilon}$ for different values of $D$. We plot the results in terms of anomalous dimensions, defined as the difference between an operator's scaling dimension and the scaling dimension of the corresponding field in the free scalar theory in $D$ dimensions:

$$
\begin{aligned}
& \gamma_{\sigma} \equiv \Delta_{\sigma}-\Delta_{\varphi}=\Delta_{\sigma}-(D-2) / 2, \\
& \gamma_{\epsilon} \equiv \Delta_{\epsilon}-\Delta_{\varphi^{2}}=\Delta_{\epsilon}-(D-2) .
\end{aligned}
$$

As expected, all the bounds possess kinks, which become sharper as $D \rightarrow 4$. These kinks are clearly special points in the space of scaling dimensions. By construction, for the $\langle\sigma \sigma \sigma \sigma\rangle$ correlator crossing symmetry has a solution anywhere below the bound. We conjecture that at the kinks this

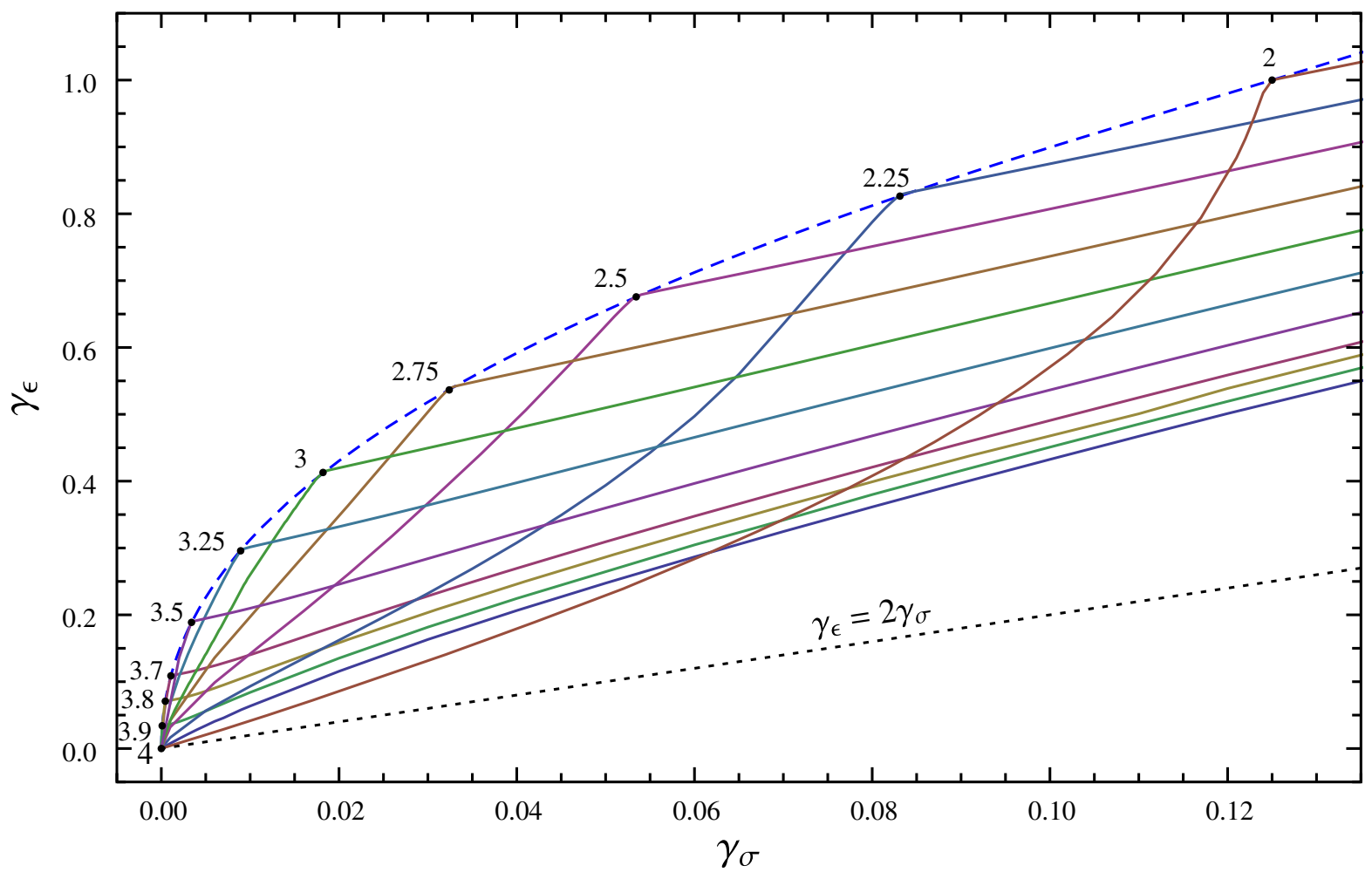

FIG. 1 (color online). Upper bounds on $\gamma_{\epsilon}$ as a function of $\gamma_{\sigma}$, plotted for $D=2,2.25, \ldots, 4$. For each $D<4$, the bound shows a kink, where a CFT belonging to the Ising model universality class is conjectured to live (black dots, fitted by the blue dashed curve). An example of theories in the bulk of the allowed region are Gaussian models, where $\gamma_{\epsilon}=2 \gamma_{\sigma}$ (black dotted line). 

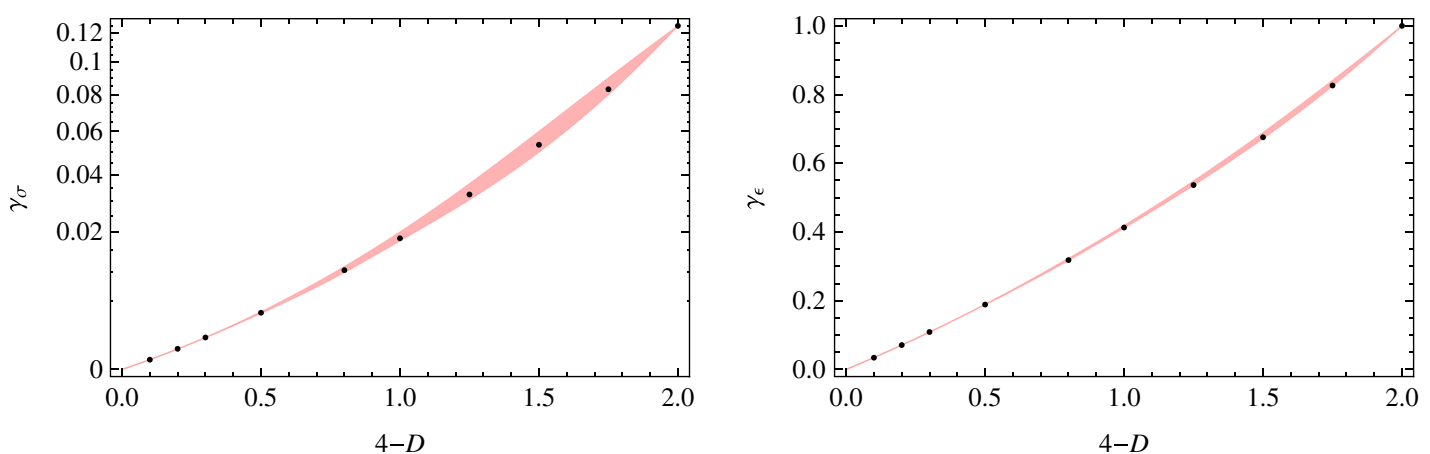

FIG. 2 (color online). Black dots: The anomalous dimensions corresponding to the kinks in Fig. 1. Red bands: The same dimensions determined by Borel-resumming the $\varepsilon$ expansion series [31]. Since $\gamma_{\sigma}=O\left(\varepsilon^{2}\right)$, we use a square root scale on the $\gamma_{\sigma}$ axis.

solution can be extended to all the correlators of the theory; i.e., there is a full-fledged CFT corresponding to these operator dimensions.

To test this conjecture, we compare the positions of the kinks with the $\varepsilon$ expansion. We use the results of Ref. [31], where the $\varepsilon$ expansion was Borel-resummed for a number of dimensions between two and four, imposing agreement with the exactly known 2D critical exponents as a boundary condition. As Fig. 2 shows, we find excellent agreement within the stated error bars [32]. For $\varepsilon \lesssim 0.5$ errors due to ambiguities in the resummation procedure are negligible, and our points precisely track the $\varepsilon$ expansion curve [33].

Central charges. - A future goal of this study is to be able to track the spectrum of the CFTs at the kinks from the free 4D scalar to the Ising model in 2D. As a preliminary step in this program, we investigate the value of the central charge, defined as the coefficient in the two-point function of the canonically normalized stress tensor. We normalize the two-point function so that the free scalar central charge is $c_{T}^{\text {free }}=D /(D-1)$ [34].

Hence, for any dimension $D$, we assume the maximal gap allowed by our dimension bound, and we extract the solution to the crossing symmetry constraint for several values of $\gamma_{\sigma}$ around the kink. For $\gamma_{\sigma}$ fixed and $\gamma_{\epsilon}$ approaching the bound from below, we observe that the dimensions and OPE coefficients of low-lying operators in the solution approach finite limits. Such a behavior was previously speculated in Refs. [4,11]; a dual version of the same phenomenon was demonstrated in the 2D case in Ref. [12]. Here we focus on the stress tensor of the theory, identified as the symmetric traceless rank-two tensor operator of dimension $D$. By inspection, an operator with such quantum numbers turns out to be always present in the limiting solution. The value $\lambda_{D, 2}^{2}$ of its OPE coefficient squared is then related to the central charge by an inverse relation:

$$
c_{T}=\left(\Delta_{\sigma}^{2} / \lambda_{D, 2}^{2}\right) c_{T}^{\text {free }},
$$

where $\lambda_{D, 2}^{2}$ is extracted by normalizing the conformal blocks as in Refs. [11,20].

For each $D$, Eq. (3) gives $c_{T}$ as a function of $\gamma_{\sigma}$, the dependence coming both from the $\Delta_{\sigma}$ in Eq. (3) and from the fact that $\lambda_{D, 2}^{2}$ is determined from the limiting solution which is a nontrivial function of $\gamma_{\sigma}$. For any $D$, the dependence of $c_{T}$ on $\gamma_{\sigma}$ is qualitatively similar to Fig. 11 in Ref. [11]. Namely, it turns out that $c_{T}$ has a minimum for $\gamma_{\sigma}$ at the kink, which we would like to identify as the value of $c_{T}$ for the CFT living at the kink [35]. For $D=2$ this agrees very precisely with the exact $2 \mathrm{D}$ Ising model value $[12,36]$. Interestingly, $c_{T}<c_{T}^{\text {free }}$ for all $2 \leq D<4$, although Zamolodchikov's $c$ theorem mandates this only for $D=2$. In Fig. 3, we plot the normalized difference $\left(c_{T}^{\text {free }}-c_{T}\right) / c_{T}^{\text {free }}$ as a function of $\varepsilon$. This represents our prediction for the central charge as the space-time dimension changes from 4 to 2 . The dashed line in the same plot shows the $\varepsilon$ expansion prediction [37-40]:

$$
\left(c_{T}^{\text {free }}-c_{T}\right) / c_{T}^{\text {free }}=5 \varepsilon^{2} / 324+\cdots .
$$

The agreement is good for $\varepsilon \lesssim 0.3$, but for larger values the unknown higher order corrections must be significant.

Discussion.-The results of this study show clearly that the family of CFTs conjectured by Wilson and Fisher can be identified at the nonperturbative level by using the conformal bootstrap. The bootstrap predictions match well

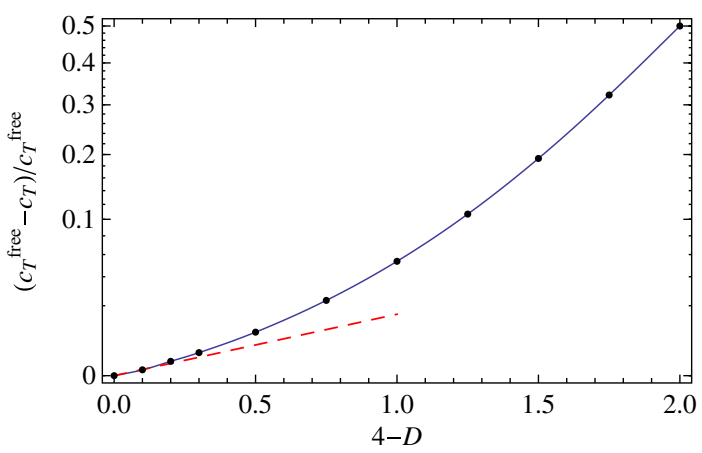

FIG. 3 (color online). Black dots: The normalized central charge difference (on a square root scale) corresponding to the kinks in Fig. 1, interpolated by the blue curve. The dashed red line is the lowest-order $\varepsilon$ expansion prediction. 
with the best estimates from the Borel-resummed $\varepsilon$ expansion for $\gamma_{\sigma}$ and $\gamma_{\epsilon}$ and greatly surpass previous perturbative computations of the central charge. We are optimistic that we will soon obtain precise bootstrap predictions in this family of CFTs for the full low-lying spectrum of operator dimensions and OPE coefficients.

There are still a number of important questions that remain to be answered-why does this family of CFTs occupy a special place in the space allowed by crossing symmetry and unitarity? Can we gain a better analytic understanding of the transitions across the kinks? Can one apply similar techniques on other correlators to learn about the $\mathbb{Z}_{2}$-odd spectrum? Finally, can one adapt similar techniques to identify and learn about CFTs living in the interior? We hope that these and related questions can be addressed in future work.

While in this Letter we studied only $2 \leq D \leq 4$, it should be straightforward and interesting to extend our analysis to $1<D<2$, where the line of Wilson-Fisher fixed points is believed to continue [31], and to connect to the conformal bootstrap studies in $D=1$ [41]. From the CFT point of view, the $D \rightarrow 1$ limit naïvely looks discontinuous, since in $D=1$ we have only one cross ratio and no spin. This issue deserves a detailed study.

S. R. is grateful to J. Cardy for useful discussions and to H. W. Diehl for informing him about Refs. [42-44]. We thank the organizers and participants for the stimulating environment at the "Back to the Bootstrap 3" conference at CERN. The work of S. E. was supported by the French ANR Contract No. 05-BLAN-NT09-573739, the ERC Advanced Grant No. 226371, and the ITN program PITN-GA-2009237920. M.P. is supported by DOE Grant No. DESC0010010-Task A. D. P. thanks the Galileo Galilei Institute for Theoretical Physics and the INFN for hospitality and partial support during the completion of this work. The work of S.R. is supported in part by the European Program "Unification in the LHC Era," Contract No. PITNGA-2009-237920 (UNILHC). The work of D. S. D. is supported by DOE Grant No. DE-SC0009988. D. S.-D. thanks SLAC for hospitality while this work was being completed. This research used resources of the National Energy Research Scientific Computing Center, which is supported, as the work of A. V., by the Office of Science of the U.S. Department of Energy under Contract No. DEAC02-05CH1123.

[1] R. Rattazzi, V. S. Rychkov, E. Tonni, and A. Vichi, J. High Energy Phys. 12 (2008) 031.

[2] V.S. Rychkov and A. Vichi, Phys. Rev. D 80, 045006 (2009).

[3] F. Caracciolo and S. Rychkov, Phys. Rev. D 81, 085037 (2010).

[4] D. Poland and D. Simmons-Duffin, J. High Energy Phys. 05 (2011) 017.
[5] R. Rattazzi, S. Rychkov, and A. Vichi, Phys. Rev. D 83, 046011 (2011).

[6] R. Rattazzi, S. Rychkov, and A. Vichi, J. Phys. A 44, 035402 (2011).

[7] A. Vichi, J. High Energy Phys. 01 (2012) 162.

[8] D. Poland, D. Simmons-Duffin, and A. Vichi, J. High Energy Phys. 05 (2012) 110.

[9] S. Rychkov, arXiv:1111.2115.

[10] P. Liendo, L. Rastelli, and B. C. van Rees, J. High Energy Phys. 07 (2013) 113.

[11] S. El-Showk, M. F. Paulos, D. Poland, S. Rychkov, D. Simmons-Duffin, and A. Vichi, Phys. Rev. D 86, 025022 (2012).

[12] S. El-Showk and M. F. Paulos, Phys. Rev. Lett. 111, 241601 (2013).

[13] C. Beem, L. Rastelli, and B. C. van Rees, Phys. Rev. Lett. 111, 071601 (2013).

[14] F. Kos, D. Poland, and D. Simmons-Duffin, arXiv:1307.6856.

[15] F. Gliozzi, Phys. Rev. Lett. 111, 161602 (2013).

[16] S. Ferrara, A. F. Grillo, and R. Gatto, Ann. Phys. (N.Y.) 76, 161 (1973).

[17] A. M. Polyakov, Zh. Eksp. Teor. Fiz. 66, 23 (1974).

[18] F. Dolan and H. Osborn , Nucl. Phys. B599, 459 (2001).

[19] F. Dolan and H. Osborn, Nucl. Phys. B678, 491 (2004).

[20] F. Dolan and H. Osborn, arXiv:1108.6194v2.

[21] M. Hogervorst and S. Rychkov, Phys. Rev. D 87, 106004 (2013).

[22] M. Hogervorst, H. Osborn, and S. Rychkov, J. High Energy Phys. 08 (2013) 014.

[23] A. L. Fitzpatrick, J. Kaplan, and D. Poland, J. High Energy Phys. 08 (2013) 107.

[24] I. Heemskerk, J. Penedones, J. Polchinski, and J. Sully, J. High Energy Phys. 10 (2009) 079.

[25] A. L. Fitzpatrick, J. Kaplan, D. Poland, and D. SimmonsDuffin, J. High Energy Phys. 12 (2013) 004.

[26] Z. Komargodski and A. Zhiboedov, J. High Energy Phys. 11 (2013) 140.

[27] K. G. Wilson and M. E. Fisher, Phys. Rev. Lett. 28, 240 (1972).

[28] K. G. Wilson, Phys. Rev. D 7, 2911 (1973).

[29] This assumption can been checked order by order in the $\varepsilon$ expansion by exhibiting the conformal symmetry algebra of the anomalous dimension matrix of composite operators, as has been done at $O(\varepsilon)$ in $[42,43]$ and at $O\left(\varepsilon^{2}\right)$ in [44].

[30] S. El-Showk, M. F. Paulos, D. Poland, S. Rychkov, D. Simmons-Duffin, and A. Vichi, arXiv:1403.4545v1.

[31] J. Le Guillou and J. Zinn-Justin, J. Phys. (Paris) 48, 19 (1987).

[32] Although the $O\left(\varepsilon^{5}\right)$ terms used in [31] contain a mistake since then corrected in [45], the induced error is negligible [46].

[33] The lowest order $\varepsilon$ expansion result for $\gamma_{\epsilon}$ was reproduced by the conformal bootstrap methods long ago in [17].

[34] H. Osborn and A. Petkou, Ann. Phys. (N.Y.) 231, 311 (1994).

[35] In fact, exactly the same values of the central charge for boundary solutions can be obtained by computing bounds on OPE coefficients as in [11].

[36] A. Vichi, Ph.D. thesis, École Polytechnique Federal Lausanne, 2011.

[37] S. J. Hathrell, Ann. Phys. (N.Y.) 139, 136 (1982). 
[38] I. Jack and H. Osborn, Nucl. Phys. B234, 331 (1984).

[39] A. Cappelli, D. Friedan, and J. I. Latorre, Nucl. Phys. B352, 616 (1991).

[40] A. Petkou, Ann. Phys. (N.Y.) 249, 180 (1996).

[41] D. Gaiotto, D. Mazac, and M. F. Paulos (to be published).

[42] S. Kehrein, F. Wegner, and Y. Pismak, Nucl. Phys. B402, 669 (1993).
[43] S. K. Kehrein and F. Wegner, Nucl. Phys. B424, 521 (1994).

[44] S. K. Kehrein, Nucl. Phys. B453, 777 (1995).

[45] H. Kleinert, J. Neu, V. Schulte-Frohlinde, K. Chetyrkin, and S. Larin, Phys. Lett. B 272, 39 (1991).

[46] R. Guida and J. Zinn-Justin, J. Phys. A 31, 8103 (1998). 